\title{
NOTE
}

\section{Fatal Erysipelothrix rhusiopathiae septicemia in two Atlantic dolphins (Stenella frontalis and Tursiops truncatus)}

\author{
J. Díaz-Delgado ${ }^{1, *}$, M. Arbelo ${ }^{1}$, E. Sierra ${ }^{1}$, A. Vela ${ }^{2}$, M. Domínguez ${ }^{3}$, Y. Paz ${ }^{1}$, \\ M. Andrada ${ }^{1}$, L. Domínguez ${ }^{2}$, A. Fernández ${ }^{1}$ \\ ${ }^{1}$ Veterinary Histology and Pathology, Institute of Animal Health, Veterinary College, \\ University of Las Palmas de Gran Canaria, Trasmontana s/n, Arucas 35413, Las Palmas de Gran Canaria, Spain \\ ${ }^{2}$ Departamento de Sanidad Animal, Facultad de Veterinaria, Universidad Complutense, Avenida Puerta de Hierro s/n, \\ 28040 Madrid, Spain \\ ${ }^{3}$ Servicio de Inmunología, Centro Nacional de Microbiología, Instituto de Salud Carlos III, Av de Monteforte de Lemos 5 , \\ 28029 Madrid, Spain
}

\begin{abstract}
We describe gross, histopathologic, ultrastructural, immunohistochemical, and microbiologic features of acute septicemia by Erysipelothrix rhusiopathiae in an Atlantic spotted dolphin Stenella frontalis and an Atlantic bottlenose dolphin Tursiops truncatus. Generalized lymphadenomegaly and widespread hemorrhages were the most consistent macroscopic findings. Tricavitary effusion and icterus were noted in one individual. Histologically, all organs examined showed numerous variably sized bacillary bacterial emboli (Gram-positive; Ziehl-Neelsen-negative), typically associated with systemic congestion, edema, hemorrhages, and fibrinocellular thrombi. These bacteria were frequently intravascular, either extracellular or intramonocytic/ macrophagic, and to a lesser extent, free within the interstitium of parenchymal organs. In both cases, microbiological analysis yielded E. rhusiopathiae. A primary anti-E. rhusiopathiae antibody created in mice from one of the strains isolated allowed positive immunohistochemical detection. Electron microscopy and dual immunohistochemistry with lysozyme and MAC387 antibodies confirmed the intramacrophagic location of the bacilli. E. rhusiopathiae, a known multispecies and zoonotic agent, should be considered as a potential etiologic agent in septicemia cases in freeranging individuals of these dolphin species.
\end{abstract}

KEY WORDS: Cetacean pathology $\cdot$ Bacteremia $\cdot$ Zoonosis $\cdot$ Natural disease $\cdot$ Immunohistochemistry · Ultramicroscopy

\section{INTRODUCTION}

Marine mammals are recognized as useful sentinels of the health status of the oceans (Bossart 2011). Due to their frequent interaction with humans, cetaceans comprise some of the most suitable species to monitor the human-marine mammal interface. Zoonoses are of major concern to marine mammal researchers, rehabilitators, trainers, veterinarians, and volunteers, as they are at increased risk of acquiring zoonotic diseases through occupational exposure. Several pathogens from marine mammals have been confirmed to infect humans, including Ajellomyces (Blastomyces) dermatitidis, Bisgaardia sp., Brucella sp., calicivirus, Erysipelothrix rhusiopathiae, influenza viruses, Lacazia (Loboa) loboi, Leptospira sp., Mycobacterium sp., Mycoplasma sp., poxvirus, and Streptococcus sp. (Waltzek et al. 2012). 
Koch first isolated a member of the genus Erysipelothrix in 1876 (Koch 1878) from mice that had been inoculated with blood from putrefied meat, and the first isolation from a pig was in 1882. The genus currently contains 3 species: E. rhusiopathiae, E. tonsillarum, and E. inopinata (Stackebrandt et al. 2006). Some investigations on the pathogenicity of E. tonsillarum have concluded that most serotypes are apathogenic, e.g. in swine (Takahashi et al. 1987) and chickens (Takahashi et al. 1994), yet certain serotypes have been incriminated as primary pathogens, e.g. in dogs (Takahasi et al. 2000) and swine (Bender et al. 2011). The pathogenicity of E. inopinata, first isolated as a contaminant from sterile-filtered vegetable broth, is largely unknown (Verbarg et al. 2004). E. rhusiopathiae has been found as a commensal or a pathogen in myriads of vertebrate and invertebrate species worldwide (Stackebrandt et al. 2006). Its major reservoir is believed to be domestic swine, but rodents and birds are also frequently infected (Wang et al. 2010).

Since first reported in a cetacean species (Seibold \& Neal 1956), Erysipelothrix infection has been recognized in up to 10 different species, including captive and free-ranging individuals (Melero et al. 2011), with descriptions of the classic dermatologic (Simpson et al. 1958) and acute septicemic forms resembling those reported in swine (Seibold \& Neal 1956, Dilbone 1965, Geraci et al. 1966, Kinsel et al. 1997). Although peracute-to-acute septicemia occurs with little or no specific clinical signs (Seibold \& Neal 1956), and diagnosis is generally made postmortem, pathognomonic diamond-shaped skin lesions are seen in the chronic form of the disease (Melero et al. 2011). For both presentations, the diagnosis relies on clinical signs and culture with selective and enrichment media, classic bacterial identification systems e.g. API Coryne System 2.0 (bioMérieux), and molecular identification. Serologic studies in cetacean populations have been limited due to lack of commercial kits available; however, use of indirect immunofluorescence (IIF), agglutinating techniques, and ELISA has allowed detection of positive anti-Erysipelothrix titers in wild dolphins (Gilmartin et al. 1971, Suer et al. 1988, Bernal-Guadarrama et al. 2014). Erysipelothrix zoonotic potential, with emphasis on the marine mammal-human interface, has been recognized for personnel involved in whaling and sealing activities with the classical 'seal or whale-finger' (Hillenbrand 1953) or necropsy operations on cetacean carcasses (Chastel et al. 1975).

Erysipelothrix infection is considered the most serious infectious disease of captive cetaceans, with unvaccinated juveniles being most susceptible (Fraser
1986). Likely sources of infection include ingestion of contaminated feedstuffs and opportunistic colonization of wounds. Current prophylactic approaches include antibiotic therapy (e.g. penicillin) and vaccination with porcine erysipelas vaccines (BernalGuadarrama et al. 2014), yet immunogenicity has been shown to vary between commercially available products (Dunn et al. 2001). Vaccination with the porcine erysipelas bacterin has resulted in adverse clinical reactions and even death (Bernal-Guadarrama et al. 2014), resulting in reduced use worldwide (Dunn et al. 2001).

The present study describes gross histopathologic, ultrastructural, immunohistochemical (IHC), and microbiologic features of acute septicemia by $E$. rhusiopathiae in a free-ranging Atlantic spotted dolphin Stenella frontalis and an Atlantic bottlenose dolphin Tursiops truncatus. To the best of our knowledge, even though this agent has been recorded in these species, neither IHC nor electron microscopy has been previously employed in cetacean Erysipelothrix infection.

\section{MATERIALS AND METHODS}

A $228 \mathrm{~cm}$ long, adult female Atlantic bottlenose dolphin (Animal 1) was submitted for necropsy after being found stranded dead in Las Galletas, Tenerife (28 0' 18' N, 16³9' 21" W; Canary Islands) in March 2010. A 42 kg (92.59 lb), 160 cm long, subadult male Atlantic spotted dolphin (Animal 2) was submitted for necropsy after being found stranded dead in the same location in February 2012. Both individuals were necropsied having a fresh decomposition code (grade 2) (Kuiken \& García-Hartmann 1991) and a good body condition (Arbelo et al. 2013). The required permission for the management of stranded cetaceans anywhere within the Canarian archipelago was issued by the environmental department of the Canary Islands' Government. No experiments were performed on live animals because our work was based on dead stranded cetaceans, and the field studies did not involve endangered or protected species.

A complete and standardized necropsy (Kuiken \& García-Hartmann 1991) was performed on both individuals. Representative samples of skin, longissimus dorsi and rectus abdominis muscles, peritoneum, diaphragm, brain, pterygoid sacs, tympanoperiotic complexes, tongue, oral mucosa, pharyngeal and laryngeal tonsils, esophagus, stomach, intestine, liver, pancreas, trachea, lungs, heart, aorta, kidneys, ure- 
ters, urinary bladder, lymph nodes, testicles, penis, prepuce, ovaries, uterus, vagina, and vulva were collected and fixed in $10 \%$ neutral buffered formalin. All these tissues were processed routinely, and embedded in paraffin, and $5 \mu \mathrm{m}$ sections were stained with hematoxylin and eosin (H\&E). Selected sections were also stained with Gram, Ziehl-Neelsen (ZN), and periodic acid-Schiff (PAS).

Samples of liver, lung, and mesenteric lymph node (Animals 1 and 2), and brain and kidney (Animal 2) were surface-plated on Columbia blood agar (bioMérieux) and incubated aerobically and under anaerobic conditions for $48 \mathrm{~h}$ at $37^{\circ} \mathrm{C}$. Isolates were identified using the commercial API Coryne system. Isolates were molecularly characterized by pulsedfield gel electrophoresis (PFGE) according to the specifications of Vela et al. (2000) with the CHEF-DR III system (Bio-Rad Laboratories). The restriction enzyme Bsp 120I (MBI Fermentans) was used according to the manufacturer's recommendations.

Transmission electron microscopy (TEM) was performed on liver sections of Animal 2. Formalin-fixed samples were postfixed in suspension with $2.5 \%$ glutaraldehyde in $0.1 \mathrm{M}$ phosphate buffer ( $\mathrm{pH} 7.2$ ), and further with osmium tetroxide, stained with $1 \%$ uranyl acetate, and embedded in epon (Epon 812, Fluka Chemie). Ultra-thin sections were cut at $50 \mathrm{~nm}$, contrasted with lead citrate, and observed under a Zeiss EM912 TEM.

For IHC, the antibodies (Ab) used were a monoclonal mouse anti-human myeloid/histiocyte antigen clone MAC387 (1:100; Dako), a polyclonal rabbit anti-human lysozyme (1:400; Dako) for macrophages, and a primary polyclonal Ab (1:100) created in mice infected with the strain of Erysipelothrix rhusiopathiae isolated from Animal 1. Female BALB/C mice were immunized every other week for 3 mo by intraperitoneal injection with $5 \times 10^{8}$ total bacterial count paraformaldehyde-fixed bacteria emulsified in incomplete Freund's adjuvant (SIGMA Aldrich). A week after the last injection, the serum $\mathrm{Ab}$ titer was determined by indirect ELISA.

\section{RESULTS}

Major gross findings in Animal 1 included diffuse subcutaneous and visceral icterus and tricavitary effusion (ascites, $700 \mathrm{ml}$, Fig. 1A; hydrothorax, $250 \mathrm{ml}$; hydropericardium, $25 \mathrm{ml}$, Fig. 1B), dorsocervical subcutaneous edema with multifocal congestive areas and scattered hemorrhages, marked congestion of epaxial musculature, generalized lymphadenome- galy, hepatic lipidosis, pulmonary congestion and atelectasis, adrenal hemorrhages, and congestion and multifocal meningeal hemorrhages (Fig. 1C). Parasites included Syncyamus sp., Xenobalanus sp., and adults of Crassicauda sp. in the margins of the blowhole, caudal margins of the dorsal fin, and subcutis and thoracolumbar fascia, respectively. In Animal 2, there were multifocal cutaneous tattoo-like lesions (presumptive dolphin poxvirus; Geraci et al. 1979) and hemorrhages, widespread pale tan-to-white irregular myocardial areas interpreted as acute rhabdomyolysis (Fig. 1D) with scattered hemorrhages, bilateral pulmonary emphysema, generalized lymphadenomegaly, and pancreatic hemorrhages. Parasites included merocercoids of Phyllobothrium delphini, adults of Crassicauda sp., Anisakis sp., Pholeter sp., unidentified cestodes, and Stenurus sp. in the integument, thoracolumbar fascia, keratinized and glandular stomach, intestine, and pterygoid sacs, respectively.

Histologically, the most consistent finding in both animals was numerous variably sized bacterial emboli throughout all tissues examined, typically associated with congestion, edema, and microhemorrhages, and less frequently with fibrinocellular thrombi. Slender, Gram-positive and ZN-negative bacilli were within blood vessels or less frequently within the interstitium of parenchymal organs. Often, intravascular bacteria were within the cytoplasm of monocytes/macrophages. In Animal 1, additional histologic findings were acute neutrophilic myocarditis (Fig. 2A), acute embolic glomerulonephritis (Fig. 2B), interstitial nephritis, embolic adrenalitis, moderateto-marked multicentric and splenic reactive lymphoid hyperplasia, lymphoplasmacytic bronchointerstitial pneumonia and alveolar nematode larvae, pyogranulomatous mesenteric lymphadenitis, granulomatous mastitis and galactophoritis with intralesional adult Crassicauda sp. and eggs, chronic lymphoplasmacytic periportal hepatitis, and perivascular cerebral, cerebellar, and spinal cord meningeal hemorrhages and edema. Animal 2 had acute cardiomyocyte degeneration and necrosis with hemorrhages, suppurative cortical adrenalitis with vascular fibrinoid necrosis, lymphocytic and histiocytic bronchointerstitial pneumonia and edema, and multicentric reactive lymphoid hyperplasia with marked sinus histiocytosis.

Gram-positive, catalase-negative, facultative anaerobic bacilli were isolated in pure culture from all samples. Isolates were identified as Erysipelothrix rhusiopathiae (API Coryne numerical code 0000340). Isolates of Animal 1 exhibited a unique PFGE profile 

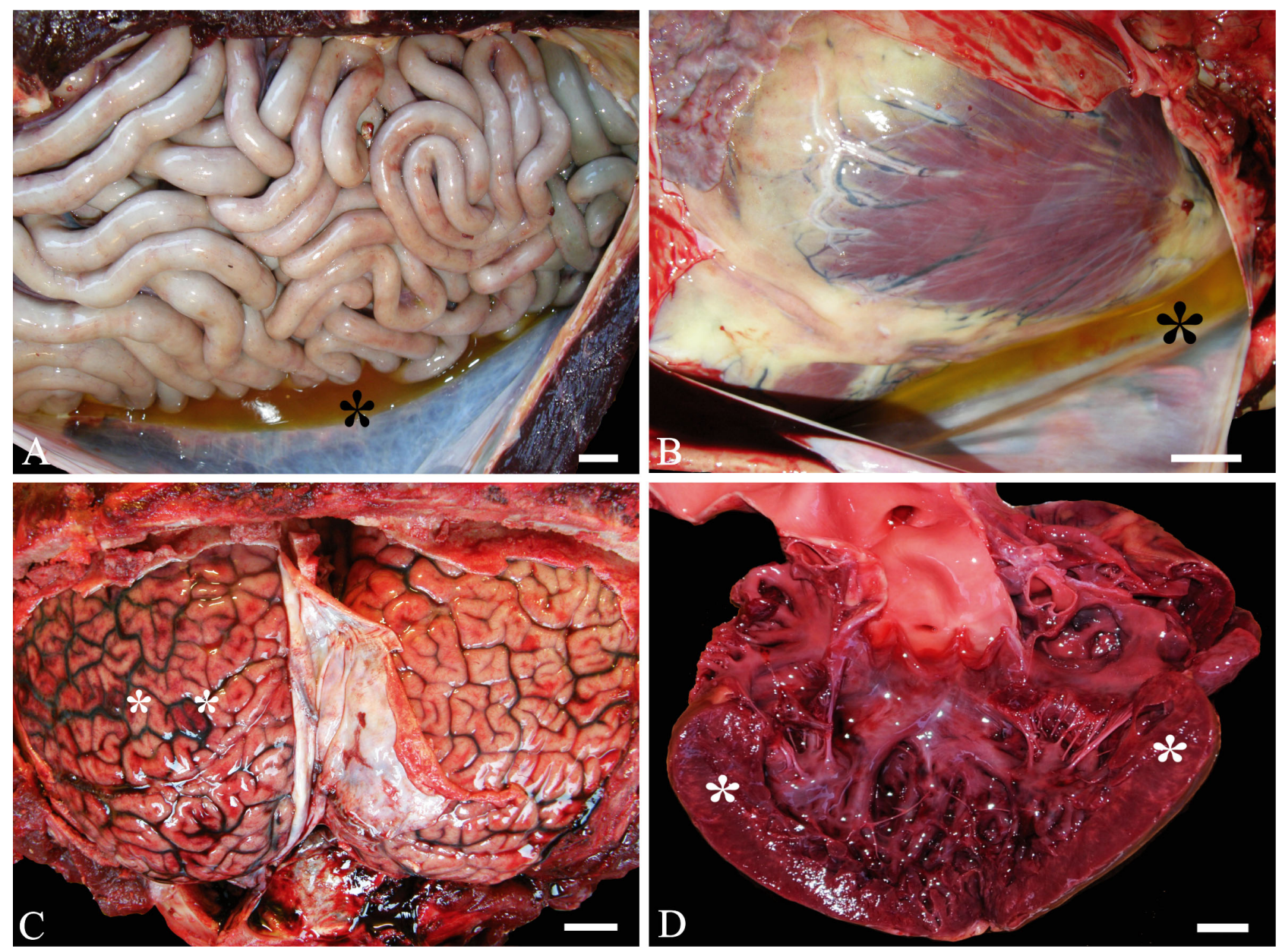

Fig. 1. Macroscopic lesions found in (A-C) Tursiops truncatus and (D) Stenella frontalis stranded dead. (A) Abdomen contains small amount of bright yellow, thin fluid (ascites, icterus) (asterisk). (B) Pericardial cavity has small amount of bright yellow, thin fluid (hydropericardium, icterus) (asterisk). (C) Bilaterally, cerebral leptomeningeal vasculature is engorged (hyperemia, congestion), more prominent in left cerebral hemisphere wherein multifocal areas of hemorrhage (asterisks) follow the vascular ramifications. Dura mater still attached at level of falx cerebri. (D) Multifocal, irregular and poorly demarcated pale areas throughout myocardium of left ventricle (asterisks), atrium, and auricle, extending from pericardium to endocardium. Scale bars $=(A) 2 \mathrm{~cm},(B-D) 1 \mathrm{~cm}$

that was different to that shown by the single PFGE profile displayed by the isolates of Animal 2, demonstrating that infections of each animal were caused by a single strain, but different E. rhusiopathiae.

In sections examined (liver, kidney) of both animals, approximately $30-50 \%$ and $75 \%$ of the intracellular and extracellular bacteria were labeled positive for anti-E. rhusiopathiae Ab, respectively (Fig. 2C). About $50-75 \%$ of the bacteria-laden macrophages showed granular cytoplasmic immunopositivity for anti-MAC387 and anti-lysozyme Ab.

Ultrastructurally, monocytes/macrophages contained small to large numbers of intracytoplasmic $2-4 \times 0.3 \mu \mathrm{m}$, straight, replicating bacilli, typically within phagosomes and phagolysosomes, or rarely free (Fig. 2D), numerous mitochondria, and lysosomes. Bacteria had osmiophilic cell walls, thin cell membranes, dense nuclear regions, and lipid storage bodies.

\section{DISCUSSION}

The cause of death in both individuals was determined as acute septicemia by Erysipelothrix rhusiopathiae infection. These cases display similar gross and microscopic findings to those reported previously in these species (Seibold \& Neal 1956, Dilbone 1965, Geraci et al. 1966, Kinsel et al. 1997). Although 2 major forms have historically been recog- 



Fig. 2. (A-C) Microscopic findings in Tursiops truncatus and (D) ultrastructural findings in Stenella frontalis, both stranded dead. (A) Multifocally, bacteria-laden monocytes and macrophages (asterisks), intravascular and interstitial extracellular (arrows) bacilli within myocardium. Mild vascular exocytosis and infiltration of neutrophils and mildly increased clear space between cardiomyocytes (edema). H\&E stain. (B) Multifocally, glomerular capillaries are dilated and filled with dense emboli of basophilic bacteria (asterisks). Rare monocytes/macrophages with engulfed bacteria (arrows). Mild increase of fibrous connective tissue around glomerular capsule and adjacent interstitium (arrowheads). H\&E stain. (C) Intramonocytic/macrophagic and extracellular bacteria labeled variably positive with anti-Erysipelothrix rhusiopathiae antibodies (asterisks). Non-commercial anti-E. rhusiopathiae antibody with antigen-specific staining with AEC substrate chromogen (red immunolabelling) and counterstained with Mayer's hematoxylin. (D) Activated macrophage with large number of intracytoplasmic bacilli typically within phagolysosomes (asterisks) or rarely free (arrows). Scale bars = (A-C) $100 \mu \mathrm{m}$, (D) $2 \mu \mathrm{m}$

nized, acute septicemic and dermatologic, there appears to be sufficient evidence to distinguish between a peracute-acute form with absent or unspecific clinical signs, e.g. anorexia, lethargy, and initial leukocytosis shortly followed by a severe leukopenia just prior to death, and subacute and chronic forms, where there is development of characteristic gray rhomboid dermal plaques secondary to dermal vasculitis with cutaneous infarction coupled with leukocytosis and anorexia (Thurman et al. 1983). In unvaccinated captive animals, individuals might survive the acute phase if appropriate treatment is instituted (Calle et al. 1993). Additionally, progression from subacute or chronic stages to acute forms have also been reported (Calle et al. 1993). Although definitive and unbiased epidemiologic data are missing, free-ranging individuals may succumb to the acute phase and this would explain the most common presentation in stranded animals. The fact that subacute or chronic forms are rarely reported in free-ranging individuals (Melero et al. 2011) may indicate that these animals are able to overcome the disease.

From 483 cetaceans stranded and subjected to necropsy examination between October 2000 and March 2015 along the coasts of the Canary Islands, 
only in the present 2 cases has Erysipelothrix infection been suspected and confirmed. A potential correlation between orographic conditions, fishery activities, or aquiculture in the stranding area and these 2 yr elapsed episodes of acute erysipelas in these 2 dolphin species is not apparent. Other possibilities would include transmission between the regional terrestrial to the local aquatic ecosystem, as has been suggested for other cetacean pathogens, e.g. Toxoplasma gondii (Bowater et al. 2003), and the recirculation of $E$. rhusiopathiae strains among these species inhabiting the area.

The pathogenesis of Erysipelothrix infection in dolphins and other species is poorly understood. Particularly in free-ranging cetaceans, information about the immunopathological features of natural disease is essentially lacking. Moreover, only a few reports have addressed in vitro immune aspects under immunization settings (Sitt et al. 2010). E. rhusiopathiae strains are known to vary considerably in virulence (Wang et al. 2010). Generally, absent specific antibodies, the organism evades phagocytosis, and even if phagocytized, it is able to replicate intracellularly. Major virulence factors investigated to date for this bacterium include hyaluronidase (a spreading factor that facilitates dissemination into tissues), neuraminidase (plays a significant role in bacterial attachment and invasion into host cells), adhesive surface proteins (e.g. RspA, RspB), and more importantly, its capsule containing the capsular polysaccharide antigen, which confers resistance to phagocytosis (Wang et al. 2010). The mechanisms mediating intracellular survival of this bacterium are unknown; superoxide dismutase production and use of certain host receptor(s) or complement receptors have been proposed (Shimoji 2000). Experimental infections in mice, pigeons, and swine have shown that phagocytosis of the bacteria was carried out primarily by macrophages, not polymorphonuclear cells (Wang et al. 2010). The prominent and widespread bacterial phagocytosis shown by monocytes and macrophages throughout all tissues examined coupled with evidences of intracellular bacterial replication support the assumption that macrophagic cells might play a major role in the pathogenesis of this bacterium in these species. Future studies will focus on the virulence determinates of strains isolated from captive and free-ranging dolphins and other immunopathological aspects of the infection.

Despite not being commonly used, lysozyme and MAC387 immunomarkers have been successfully employed in paraffin wax-embedded tissues of different cetacean species (Kumar \& Cowan 1994, Jaber et al. 2003). Likewise, ultrastructural analysis has proven to be an accurate complementary laboratorial technique allowing for the demonstration of changes at the cellular and subcellular level in disease by pathogenic prokaryotes (Brodgen 2009). In the present case, TEM afforded the observation, for the first time in a cetacean species, the consistent phagosomic and phagolysosomic location of the bacteria with minimal debris, suggesting that the bacteria largely overcame the hosts' digestive cellular apparatus.

Our results demonstrate the suitability of the combined use of an anti-E. rhusiopathiae antibody created in mice, lysozyme and MAC387 (commercially available) immunomarkers, and TEM aiding in diagnosis of E. rhusiopathiae infection in dolphins. Further analyses to ascertain the similarity of strains between potential reported regional human and domestic animal cases are warranted to better understand the ecopathology of this bacterium. This pathogen must be considered as a differential diagnosis for septicemia, stranding, and death in these species.

Acknowledgements. We thank Dr. B. Porter for valuable comments provided to improve the quality of this communication. We declare no potential conflicts of interest with respect to the research, authorship, and/or publication of this article. This study is part of a $\mathrm{PhD}$ program supported by the Ministry of Education of Spain through an FPU grant. Additional funding comes from the following grants: 123 National Project CGL2012-39681 (Subprograma BOS); 124 Regional Project SolSub C200801000288 and ProID 20100091.

\section{LITERATURE CITED}

Arbelo M, de los Monteros AE, Herráez P, Andrada M and others (2013) Pathology and causes of death of stranded cetaceans in the Canary Islands (1999-2005). Dis Aquat Org 103:87-99

Bender JS, Irwin CK, Shen HG, Schwartz KJ, Opriessnig T (2011) Erysipelothrix spp. genotypes, serotypes, and surface protective antigen types associated with abattoir condemnations. J Vet Diagn Invest 23:139-142

- Bernal-Guadarrama MJ, Garcia-Parraga D, FernandezGallardo N, Zamora-Padron R and others (2014) Development of an indirect immunofluorescence technique for the evaluation of generated antibody titers against Erysipelothrix rhusiopathiae in captive bottlenose dolphins (Tursiops truncatus). Arch Microbiol 196:785-790

> Bossart GD (2011) Marine mammals as sentinel species for oceans and human health. Vet Pathol 48:676-690

Bowater RO, Norton J, Johnson S, Hill B, O'donoghue P, Prior H (2003) Toxoplasmosis in Indo-Pacific humpbacked dolphins (Sousa chinensis) from Queensland. Aust Vet J 81:627-632

Brodgen KA (2009) Cytopathology of pathogenic prokaryotes. In: Cheville NF (ed) Ultrastructural pathology: the comparative cellular basis of disease. Wiley-Blackwell, Ames, IA, p 424-523 
Calle PP, Kenny DE, Cook RA (1993) Successful treatment of suspected erisypelas septicemia in a beluga whale (Delphinapterus leucas). Zoo Biol 12:483-490

Chastel C, Balovet G, Lucas A (1975) L'étudiant, le cétacé et le rouget, une petite épidémie d'erysipeloïde après dépeçage d'un globicéphale. Nouv Presse Med 4: 1803-1805

Dilbone RP (1965) Erysipelas suspected in two porpoises. J Am Vet Med Assoc 147:1085

Dunn JL, Buck JD, Robeck TR (2001) Bacterial diseases of cetaceans and pinnipeds. In: Dierauf LA, Gulland FMD (eds) CRC handbook of marine mammal medicine. CRC Press, Boca Raton, FL, p 309-335

Fraser CM (1986) The Merck veterinary manual. Merck \& Co., Rahway, NJ

Geraci JR, Sauer RM, Medway W (1966) Erysipelas in dolphins. Am J Vet Res 27:597-606

- Geraci JR, Hicks BD, St Aubin DJ (1979) Dolphin pox: a skin disease of cetaceans. Can J Comp Med 43:399-404

> Gilmartin WG, Allen JF, Ridgway SH (1971) Vaccination of porpoises (Tursiops truncatus) against Erysipelothrix rhusiopathiae infection. J Wildl Dis 7:292-295

Hillenbrand FKM (1953) Whale finger and seal finger their relation to erysipeloid. Lancet 261:680-681

> Jaber JR, Fernández A, Herráez P, de los Monteros AE and others (2003) Cross-reactivity of human and bovine antibodies in striped dolphin paraffin wax-embedded tissues. Vet Immunol Immunopathol 96:65-72

Kinsel MJ, Boehm JR, Harris B, Murnane RD (1997) Fatal Erysipelothrix rhusiopathiae septicemia in a captive Pacific white-sided dolphin (Lagenorhyncus obliquidens). J Zoo Wildl Med 28:494-497

Koch R (1878) Untersuchungen über die Ätiologie der Wundinfektionskrankheiten. Vogel, Leipzig

Kuiken T, García-Hartmann M (1991) Proc 1st ECS workshop on cetacean pathology: dissection techniques and tissue sampling, Saskatoon. European Cetacean Society Newsletter 17 (Spec Issue)

Kumar D, Cowan DF (1994) Cross-reactivity of antibodies to human antigens with tissues of the bottlenose dolphin, Tursiops truncatus, using immunoperoxidase techniques. Mar Mamm Sci 10:188-194

Melero M, Rubio-Guerri C, Crespo JL, Arbelo M and others (2011) First case of erysipelas in a free-ranging bottlenose dolphin (Tursiops truncatus) stranded in the Mediterranean Sea. Dis Aquat Org 97:167-170

Seibold HR, Neal JE (1956) Erysipelothrix septicemia in the porpoise. J Am Vet Med Assoc 128:537-539

Shimoji Y (2000) Pathogenicity of Erysipelothrix rhusio-

Editorial responsibility: Michael Moore,

Woods Hole, Massachusetts, USA pathiae: virulence factors and protective immunity. Microbes Infect 2:965-972

- Simpson CF, Wood FG, Young F (1958) Cutaneous lesions on a porpoise with erysipelas. J Am Vet Med Assoc 133: 558-560

Sitt T, Bowen L, Blanchard MT, Gershwin LJ and others (2010) Cellular immune responses in cetaceans immunized with a porcine erysipelas vaccine. Vet Immunol Immunopathol 137:181-189

Stackebrandt E, Reboli AC, Farrar WE (2006) The genus Erysipelothrix. In: Dworkin M, Falkow S, Rosenberg E, Schleifer KH, Stackebrandt E (eds) The prokaryotes. Springer, New York, NY, p 492-510

Suer LD, Vedros NA, Schroeder JP, Dunn JL (1988) Erysipelothrix rhusiopathiae. II. Enzyme immunoassay of sera from wild and captive marine mammals. Dis Aquat Org 5:7-13

Takahashi T, Fujisawa T, Benno Y, Tamura Y and others (1987) Erypiselothrix tonsillarum sp. nov., isolated from tonsils of apparently healthy pigs. Int J Syst Bacteriol 37 : 166-168

> Takahashi T, Takagi M, Yamaoka R, Ohishi K, Norimatsu M, Tamura Y, Nakamura M (1994) Comparison of the pathogenicity for chickens of Erysipelothrix rhusiopathiae and Erysipelothrix tonsillarum. Avian Pathol 23:237-245

Takahasi T, Fujisawa T, Yamamoto K, Kijima M, Takahashi T (2000) Taxonomic evidence that serovar 7 of Erysipelothrix strains isolated from dogs with endocarditis are Erysipelothrix tonsillarum. J Vet Med B 47:311-313

Thurman GD, Downes SJ, Fothergill MB, Goodwin NM, Hegarty MM (1983) Diagnosis and successful treatment of subacute erysipelas in a captive dolphin. J S Afr Vet Assoc 54:193-200

Vela AI, Vazquez J, Gibello A, Blanco MM and others (2000) Phenotypic and genetic characterization of Lactococcus garvieae isolated in Spain from lactococcosis outbreaks and comparison with isolates of other countries and sources. J Clin Microbiol 38:3791-3795

Verbarg S, Rheims H, Emus S, Frühling A, Kroppenstedt R, Stackebrandt E, Schumann P (2004) Erysipelothrix inopinata sp. nov., isolated in the course of sterile filtration of vegetabile peptone broth and description of Erysipelothrixaceae fam. nov. Int J Syst Evol Microbiol 54:221-225

> Waltzek TB, Cortes-Hinojosa G, Wellehan JF Jr, Gray GC (2012) Marine mammal zoonoses: a review of disease manifestations. Zoonoses Public Health 59:521-535

- Wang Q, Chang BJ, Riley TV (2010) Erysipelothrix rhusiopathiae. Vet Microbiol 140:405-417

Submitted: March 2, 2015; Accepted: June 25, 2015

Proofs received from author(s): September 1, 2015 